\title{
Ultraschall-Ausbildung für Studierende in der Schweiz
}

\author{
Roman Haria, Andrea Meienberg ${ }^{b}$, Dr. med. Luciano Braun ${ }^{c}$ \\ ${ }^{a}$ Dr. med., MME, Leiter Lehre, Berner Institut für Hausarztmedizin, Universität Bern; ${ }^{\text {b }}$ Dr. med., MME, Medizinische Poliklinik, Universität Basel; \\ ${ }^{c}$ Dr. med., Leiter Amikale Runde SGUM-Universitäten, Past President SGUM
}

\section{Ausgangslage}

Die Ultraschallausbildung verschiebt sich von der Weiterbildung zusehends in die Ausbildung - also in die präklinischen und klinischen Jahre des Medizin-Studiums. Früh in ihrer Karriere sehen sich junge Ärztinnen und Ärzte mit der Aufgabe konfrontiert, einfache Ultraschallanwendungen selbst durchzuführen [1]. Entsprechend besteht vonseiten der Studierenden ein grosses Interesse, sich in der diagnostischen Sonografie ausbilden zu lassen [2].

Bis vor wenigen Jahren gab es an Schweizer Universitäten ausschliesslich optionale Ultraschall-Kurse, welche jeweils sehr rasch überbucht waren [3]. Der 2018 neu eingeführte Lernzielkatalog des Schweizer Medizinstudiums («PROFILES») verlangte erstmals, dass Studierende "einfache Ultraschallanwendungen» praktisch durchführen können [4]. In der Folge entstanden an verschiedenen Hochschulen unterschiedliche Projekte im Bereich der Ultraschallausbildung - oft unter sehr engem Einbezug der Studierenden [3].

Parallel reagierte auch die Schweizerische Gesellschaft für Ultraschall in der Medizin (SGUM) auf die Entwicklung, u.a. mit der Schaffung einer Sektion «Young Sonographers» im Juni 2017, welche die Interessen der Studierenden und Assistenzärzten und -ärztinnen innerhalb der Gesellschaft vertritt.

\section{Weitere an dieser Publikation beteiligte Personen}

- Dr. med. Christiane Arnold Ferrari, MME, Notfallstation HFR Tafers, Universität Freiburg

- Dr. med. André Dietschi, Präsident, Schweizerische Gesellschaft für Ultraschall in Medizin (SGUM)

- Dr. med. Beat Dubs, Vorstand, Sektion Bewegungsapparat SGUM

- Dr. med. Natascha Lier, Lehrkoordinatorin, Insititut für Anatomie, Universität Zürich

Prof. Dr. Jean-Yves Meuwly, Service de radiodiagnostic et radiologie interventionnelle,

Université de Lausanne

- Dr. med. Jürg Prim, Schweizerische Gesellschaft für Ultraschall in Medizin (SGUM)

- Prof. Dr. med. Marga Rominger, Institut für diagnostische und interventionelle Radiologie, Universität Zürich

Dr. med. Frédéric Rouyer, Urgences, Université de Genève

- Med. pract. Ann-Sophie Schlager, Junior Präsidentin, Young Sonographers SGUM

Prof. Dr. Andreas Serra, Senior Präsident, Young Sonographers SGUM

- Dr. med. Hervé Spechbach, unité d'urgences ambulatoires, Université de Genève

- Prof. Dr. Rolf Wyttenbach, Radiologia, Università della Svizzera italiana
Mit der sogenannten «Amikalen Runde» wurde eine Arbeitsgruppe mit Vertretenden von Universitäten sowie der SGUM gebildet, um die Kooperation zu fördern und eine möglichst hohe Qualität der Ausbildung zu gewährleisten. Dieser Artikel skizziert die Arbeit und die Resultate dieser Arbeitsgruppe.

\section{Methodik}

Zwischen Dezember 2017 und Juli 2019 fanden in Zürich drei halbtägige Treffen der "Amikalen Runde» statt, zu welchen Vertretende der SGUM sowie aller Schweizer Universitäten eingeladen waren. Im Verlauf der Gespräche wurden die bestehenden Kurse an den einzelnen Universitäten sowie angelaufene Innovationen diskutiert. Verschiedene Kooperationen entstanden direkt aus Kontakten in dieser Gruppe. Im weiteren Verlauf formte sich auch ein Konsens zu gemeinsamen Zielen in der Ausbildung der Schweizer Studierenden in Sonografie.

\section{Resultate}

Unter Mitarbeit der Autorinnen und Autoren dieses Berichts wurden von Vertretenden der SGUM sowie der Universitäten folgende generelle Grundhaltungen vereinbart:

- Die SGUM sowie die Universitäten befürworten den Beginn der Ultraschall-Ausbildung bereits während des Studiums, z.B. als Ergänzung der Anatomie-Ausbildung

- Die Kurse sollten allen Studierenden die Mindestanforderungen der Lernziele gemäss PROFILES vermitteln und besonders interessierten Studierenden zusätzliche Möglichkeiten zur Vertiefung bieten

- Die Studierenden sind als primäre Adressaten der Bemühungen in der Konzeption der Lehrformate eng miteinzubeziehen - idealerweise auch in der Funktion von «Peer-Tutoren»

- Zur Vereinheitlichung und besseren Verzahnung mit der Weiterbildung sollen bereits im Studium SGUM-akkreditierte Ausbildungsgänge in Ultraschall angestrebt werden 


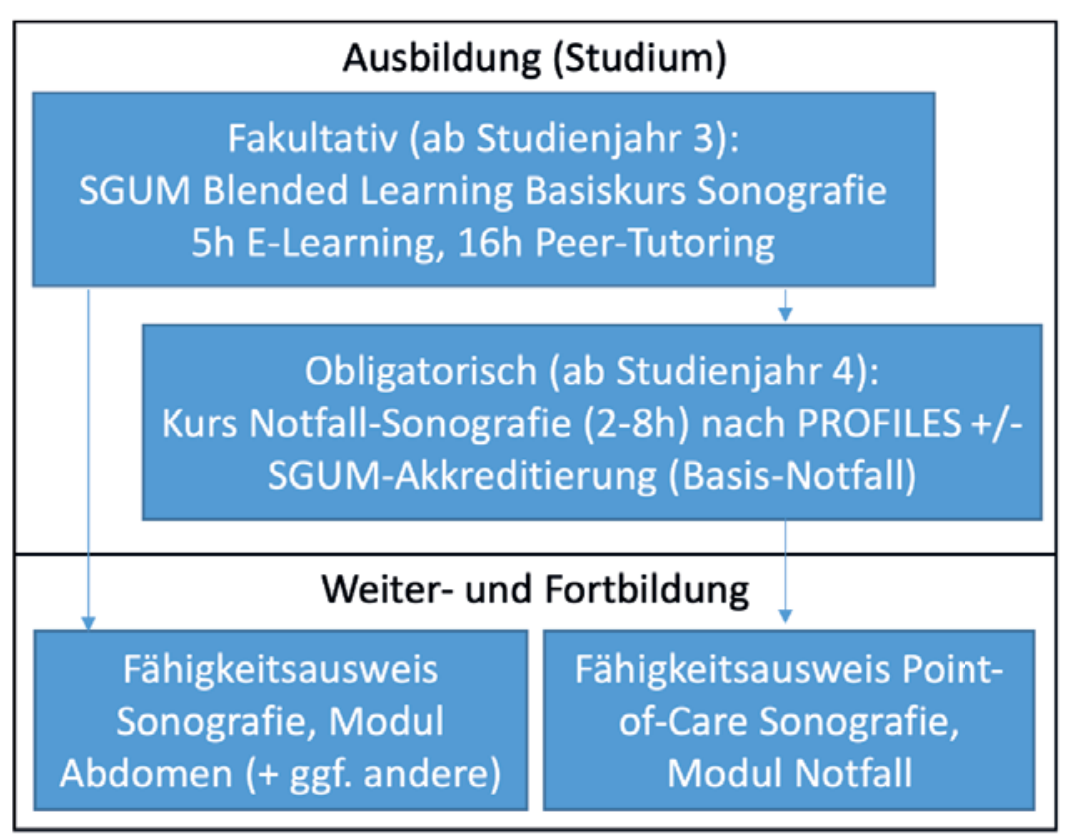

Abbildung 1: Ziel und angestrebte Kursformate der Ultraschallausbildung im Studium (Konsens).
«Basis-Notfall-Kurs» (8h) sowie 200 durchgeführte Ultraschall-Untersuchungen.

An der Universität Basel wurde 2018/2019 ein Pilotprojekt gestartet, in welchem dieser «Basis-Notfall-Kurs» an zwei Halbtagen à 4 Stunden als obligatorischer Kurs für alle Studierenden im 4. Studienjahr unterrichtet wurde. Hierbei wurden die Themen Nieren/ableitende Harnwege, Gallenblase/Gallenwege, Aorta, E-FAST, TVT und Punktionen in praktischen Kursen gelehrt, jeweils eingeführt von einem kurzen theoretischen Input. Dem praktischen Kurs vorangestellt war ein E-learning-Modul, mit einer theoretischen Einführung zur Sonografie als Methode. Die praktischen Übungen wurden von SGUM zertifizierten Tutoren und studentischen «Peer-Tutoren» angeleitet. Insgesamt absolvierten 178 Studierende den obligatorischen Kurs. Die Evaluation des Kurses konnte zeigen, dass der Kurs insgesamt sehr gut bei den Studierenden angekommen ist (5.1/6 Punkten [SD0.4]) und die Kompetenz der studentischen Tutoren als hoch gewertet wurde (5.8/7 Punkten [SD1.1]). Nach dieser erfolgreichen Pilotphase ist dieser Kurs nun fest im Basler Curriculum verankert.

Unter den bestehenden und neu konzipierten Kursen befanden sich verschiedene fakultative Kurse von 2-30 Stunden Dauer, hauptsächlich zu Inhalten der Abdomensonografie und Notfallsonografie, angeboten u.a. durch Anatomie, Notfallmedizin, Hausarztmedizin und Radiologie. Bezüglich der konkreten Kurse einigte sich die Gruppe auf folgende Ziele (s. Abb. 1):

- Angebot eines obligatorischen Kurses von mindestens zwei Stunden Dauer für alle Studierenden im Bereich der Notfall-Anwendungen zur Erfüllung des Lernziels aus PROFILES. Anzustreben ist der schrittweise Ausbau zum SGUM-anerkannten, acht-stündigen «Basis-Notfall-Kurs».

- Angebot und Förderung eines fakultativen, SGUManerkannten "Basiskurs Sonografie» an allen Universitäten für Studierende, welche sich weiter in die Anwendung der Sonografie vertiefen wollen (21h). Von Seiten der Universitäten ist hier insbesondere die Ausbildung von Peer-Tutoren sowie die Bereitstellung von Geräten und Räumen zum Selbststudium prioritär.

\section{Beschreibung der zwei geförderten Kurse:}

«Basis-Notfall-Kurs»

Im 2018 neu eingeführten Fähigkeitsprogramm «Pointof-Care» Sonografie wurde u.a. ein Fähigkeitsausweis für fokussierte Notfallsonografie geschaffen. Bedingungen für den Erwerb sind ein absolvierter «Grundkurs Abdomen/Basiskurs Sonografie» (21h), ein zusätzlicher

\section{«Blended Learning Basiskurs Sonografie»}

Unter der Leitung des Berner Instituts für Hausarztmedizin (BIHAM) wurde zwischen Mitte 2017 bis Dezember 2018 ein neues Kurskonzept entwickelt, welches den bisherigen «Grundkurs Abdomen» der SGUM abbildet, ersetzt und zum neuen «Blended Learning Basiskurs Sonografie» umwandelt. Mittelfristig soll dieser Kurs nicht nur für das «Modul Abdomen», sondern auch für die anderen Module des Fähigkeitsausweises Sonografie als gemeinsamer «Basiskurs» akkreditiert werden. Die Studierenden absolvieren dabei über einen Zeitraum von einigen Monaten nach eigener Verfügbarkeit insgesamt fünf E-Learning Module (à 1h) und 16 Stunden praktische Ultraschallausbildung im Peer-Tutoring (s. Abb. 2). Lokale studentische Gruppen organisieren sich hierfür als Untersektionen der Young Sonographers und lassen sich zu «Peer-Tutoren» ausbilden. Voraussetzung um als Peer-Tutor andere Studenten zu unterrichten ist ein abgeschlossener Abdomen Ultraschallgrundkurs und der Besuch eines didaktisch orientierten Zusatzkurses von 3 Tagen.

Der Nachweis aller absolvierter Unterrichtsstunden berechtigt die Teilnehmenden zur Anmeldung für die Abschlussprüfung. Beim Erfüllen der Mindestpunktzahl aus den 6 praktischen Ultraschall-Posten wird das Kurszertifikat «Grundkurs Abdomensonographie SGUM» ausgestellt, andernfalls muss die Prüfung nach zusätzlichem Training erneut wiederholt werden. 


\section{Blended Learning Basiskurs Sonografie}

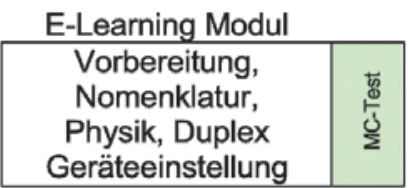

Praktische Übungsstunden (Peer Tutoren)
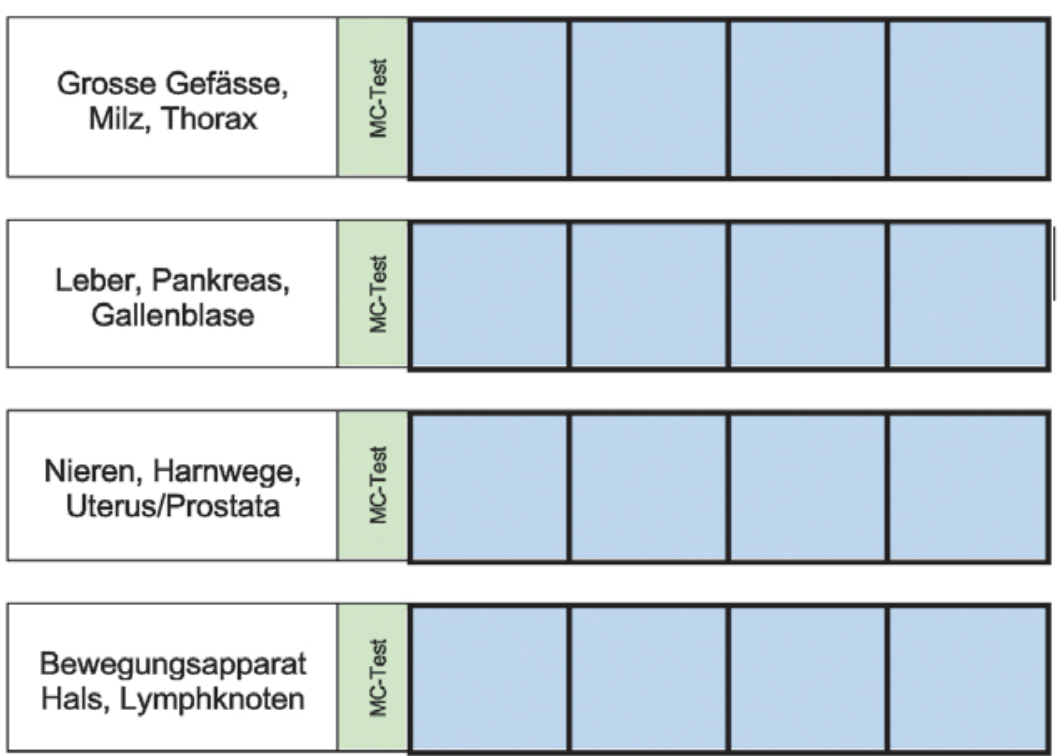

Praktische Abschlussprüfung (45min)

Abbildung 2

Dr. med. Roman Hari, MME Berner Institut für Hausarztmedizin (BIHAM)

Universität Bern Mittelstrasse 43 CH-3012 Bern Tel: +41316315873 roman.hari[at] biham.unibe.ch
Die Vorteile des Programms liegen in der zeitlichen Verteilung der Lehrstunden über einen längeren Zeitraum («spaced repetition»), dem lernfördernden Effekt der Abschlussprüfung und dem Multiplikationseffekt der verwendeten Formate. Hervorzuheben ist in diesem Modell der Bedarf der Studierenden nach zugänglichen Räumlichkeiten und Ultraschallgeräten für das Peer-Tutoring und eine gute Aus- und Weiterbildung der Peer-Tutoren durch die lokalen Universitäten. Stand Dezember 2019 haben Studierende in Bern, Zürich, Freiburg und Basel die Ausbildung im Peer Tutoring begonnen, eine Übersetzung des E-learnings auf
Französisch und ein Start in Lausanne und Genf ist auf Anfang 2020 geplant.

\section{Diskussion}

Die vorliegenden Empfehlungen zielen auf eine hohe Ausbildungs-Qualität und eine optimale Vernetzung der Aus- und Weiterbildung in der Sonografie ab. Durch das Hinarbeiten auf je einen obligatorischen und einen fakultativen, SGUM-anerkannten Kurs an allen Schweizer Universitäten kann ein hoher Grad der Standardisierung und der Kompatibilität der einzelnen Ausbildungsgänge gewährleistet werden.

Durch bestehende Kurse und verschiedene lokale Gegebenheiten sehen sich die Universitäten mit sehr unterschiedlichen Ausgangslagen konfrontiert. Entsprechend sind die Empfehlungen nicht als verbindliche Vorgaben, sondern als gemeinsame Ziele zu verstehen, welche unter der Berücksichtigung der lokalen Gegebenheiten verfolgt werden. Um den Studierenden eine Akkreditierung im Hinblick auf die Weiterbildung und spätere Tätigkeiten zu ermöglichen, ist ein gemeinsamer Standard und eine enge Zusammenarbeit mit der SGUM essentiell.

Literatur

1 Ma IWY, et al. Internal Medicine Point-of-Care Ultrasound Curriculum: Consensus Recommendations from the Canadian Internal Medicine Ultrasound (CIMUS) Group. Journal of general internal medicine. 2017;32(9):1052-7.

2 Kessler C, Bhandarkar S. Ultrasound training for medical students and internal medicine residents - a needs assessment. J Clin Ultrasound. 2010;38(8):401-8.

3 Räschle N. Narrative Review Ultraschall-Ausbildung im Medizinstudium. Masterthese Humanmedizin, Universität Bern, 2018.

4 Michaud PA, J.P., PROFILES - Principal Relevant Objectives and Framework for Integrated Learning and Education in Switzerland. Joint Commission of the Swiss Medical Schools, 2017.

Bildnachweise

Abbildungen 1 und 2: Roman Hari

\section{Interessenverbindungen}

Roman Hari ist als Projektleiter im «Blended Learning Basiskurs Sonografie» tätig. Es bestehen keine finanziellen Interessensverbindungen.

\section{Das Wichtigste in Kürze}

- Ultraschallkenntnisse werden für angehende Ärztinnen und Ärzte immer wichtiger und die Ultraschall Grundausbildung verschiebt sich entsprechend von der Weiter- in die Ausbildung der Studierenden.

- Eine Arbeitsgruppe mit Vertretern von Universitäten sowie der Schweizerischen Gesellschaft für Ultraschall in der Medizin (SGUM) hat einen Consensus für die Ausbildung im Studium erarbeitet.

- Gefördert und akkreditiert werden insbesondere der «Basiskurs Sonografie» im Blended Learning mit PeerTutoring sowie der "Notfall-Basiskurs».

- Neue Kurse sollen auf bestehenden Kursformaten aufbauen und die lokalen Gegebenheiten berücksichtigen.

\section{L'essentiel en bref}

- Les connaissances en ultrasonographie deviennent de plus en plus importantes pour les futurs médecins et la formation initiale correspondante est donc déplacée de la formation postgraduée à la formation prégraduée.

- Un groupe de travail composé de représentants des universités et de la Société suisse d'ultrasons en médecine (SSUM) a élaboré un consensus pour la formation dans les études.

- Le "Cours de base sonographie» en Blended Learning avec tutorat par les pairs et le "Cours de base urgences" sont notamment soutenus et accrédités.

- Les nouveaux cours doivent reposer sur les formats existants et tenir compte des spécificités locales. 